\title{
COMPARACIÓN DE LAS TÉCNICAS LIKERT Y DIFERENCIAL SEMÁNTICO PARA VALORAR LAS ACTITUDES HACIA LA QUÍMICA DE INGRESANTES A CARRERAS BIOLÓGICAS
}

\author{
María Silvina Reyes ${ }^{1}$ \\ sreyes@gigared.com \\ Silvia Porro ${ }^{2}$ \\ sporro@unq.edu.ar \\ María Elida Pirovani ${ }^{3}$ \\ mpirovan@fiq.unl.edu.ar
}

\section{Resumen}

Este trabajo presenta una comparación entre los cuestionarios según la Escala Likert y el método de diferencial semántico, con el fin de evidenciar si ambas técnicas pueden medir en forma equiparable la actitud hacia la química de ingresantes a las carreras de Profesorado en Biología y Licenciatura en Biodiversidad. Los resultados indican que estos instrumentos no detectan las mismas actitudes hacia la química y esto se comprueba al analizar la concordancia entre ambos, la cual es prácticamente nula.

Palabras clave

Escala Likert, diferencial semántico, actitud, concordancia, química, ingresantes.

1 Universidad Nacional del Litoral, Facultad de Humanidades y Ciencias.

2 Universidad Nacional de Quilmes, Departamento de Ciencia y Tecnología.

3 Universidad Nacional del Litoral, Facultad de Ingeniería Química. 


\section{Abstract}

This work presents a comparison between the questionnaires according to the scale Likert and semantic differential method, in order to reveal if both techniques can be measured in comparable form attitudes towards chemistry of freshmen to the careers of teachers in biology and Bachelor's degree in biodiversity. The results indicate that both instruments will not detect the same attitudes towards chemistry and this is checked to analyze the correlation between both methods, which is practically null.

\section{Introducción}

Actualmente se considera a la educación como un proceso de transformación integral de la persona, en el cual es importante comprender las variables psicológicas implicadas que permiten desarrollar la capacidad de ser, conocer e interactuar. En este sentido, es bien sabido que existen mecanismos racionales que nos dirigen, pero también emocionales que tienen un papel vital en el proceso de enseñanza y aprendizaje (Nieswandt, 2007). Autores como Duit y Treagust (2012) indican que lo cognitivo configura lo afectivo y lo afectivo lo cognitivo, por tanto, la idea de la enseñanza como una práctica emocional en la que intervienen procesos cognitivos y afectivos es aceptada por investigadores y educadores. En acuerdo con lo expresado anteriormente, es importante conocer con qué actitud se acercan los estudiantes a disciplinas científicas. La palabra actitud es sin dudas un término polisémico, que puede presentar diferentes interpretaciones. Una actitud puede definirse como la suma total de inclinaciones, sentimientos, prejuicios, nociones preconcebidas, temores o convicciones del individuo acerca de un asunto determinado (Xu y Lewis, 2011). Las actitudes tienen un carácter multidimensional que integra componentes cognitivos, afectivos y conductuales. Diversos autores sostienen que ellas pueden ser determinantes de los aprendizajes y, como pueden ser aprendidas y modificadas, son susceptibles de constituirse en objetivos a lograr. Se han realizado trabajos referidos a las actitudes de los estudiantes hacia las ciencias naturales en general y hacia la química en particular (Bauer, 2008; Garritz, Rueda, Robles y Vázquez-Alonso, 2011); sin embargo, no se han encontrado publicaciones referidas a actitudes frente a la química de estudiantes universitarios que cursan carreras netamente biológicas en Argentina.

A lo largo del tiempo se han desarrollado varios instrumentos para medir en forma cuantitativa las actitudes de los estudiantes, sin embargo, Venturini (2004) reporta que los cuestionarios más utilizados en trabajos que tienen como objetivo investigar la actitud de los estudiantes hacia las ciencias experimentales son los cuestionarios que utilizan la escala de Likert (L) y el método de diferencial semántico (DS). En el primer caso, se trata de un conjunto de reactivos de actitud, donde todos los reactivos 0 proposiciones declarativas (PD) son considerados con un valor de actitud aproximadamente igual, y donde cada uno de los participantes señala su grado de acuerdo o desacuerdo. Está formada por un conjunto de ítems presentados en forma de afirmaciones o juicios ante los cuales los sujetos tienen que manifestarse. Se trata de una escala aditiva (de ahí el nombre de escala de evaluaciones sumadas), lo que significa que las puntuaciones de los reactivos de dicha escala se suman para producir una puntuación de la actitud del individuo. El cuestionario según el método de DS consiste en una combinación de asociaciones controladas y procedimientos de escala. Se proporciona al sujeto un concepto para que sea diferenciado y un conjunto de escalas de adjetivos bipolares y/o acciones contrarias, para que lo realice; siendo su única tarea indicar para cada ítem la dirección de su asociación y su intensidad sobre una escala numérica. Existen pocos trabajos que comparan ambas técnicas ( $L$ versus DS). Espinosa García y Román Galán (1998) han encontrado que ambas técnicas de evaluación de actitudes hacia las ciencias experimentales proporcionan resultados equiparables en el sistema educativo español. Sin embargo, Odetti, Tiburzi, Mondino y Güemes (2009) concluyen que ambas técnicas no son intercambiables al evaluar las actitudes hacia la química de estudiantes 
de las carreras de Bioquímica y Licenciatura en Nutrición, a través del índice kappa de Cohen. Este índice mide el grado de acuerdo entre dos mediciones. Las mismas pueden corresponder a dos evaluadores diferentes como así también a diferentes instrumentos de evaluación, este es el caso presentado en esta investigación.

\section{Problema}

Los currículos de las carreras de Profesorado en Biología (PB) y Licenciatura en Biodiversidad (LB) que se dictan en la Universidad Nacional del Litoral (Santa Fe, Argentina) tienen a la asignatura Química General e Inorgánica (QGI) como obligatoria en el primer cuatrimestre de primer año. Por lo general, el rendimiento de los estudiantes en este primer curso de química es bajo y es común que algunos alumnos recursen dicha asignatura, lo cual claramente va en desmedro de su rendimiento académico, además de ser un factor desalentador y hasta frustrante para los estudiantes. Lo anterior reviste importancia ya que la universidad antes mencionada trabaja fuertemente en la implementación de políticas de retención y permanencia de los ingresantes en las distintas carreras que se dictan en ella. Es por esto que conocer con qué actitud se acercan los alumnos a la química permitirá encontrar nuevas estrategias para motivar a los mismos, facilitando así un aprendizaje significativo.

\section{Objetivo general}

Identificar las actitudes hacia la química de alumnos ingresantes a carreras biológicas (Licenciatura en Biodiversidad y Profesorado en Biología).

\section{Objetivos específicos}

- Evaluar la actitud frente a la química de alumnos de las carreras de Licenciatura en Biodiversidad y Profesorado en Biología, identificando si son positivas, indiferentes o negativas.

- Determinar si los métodos de diferencial semántico y escala Likert son equiparables en la medición de actitudes hacia la química.

\section{Metodología}

La metodología utilizada en esta investigación fue de tipo cuantitativa.

\section{Muestra}

Se encuestó a la totalidad de alumnos de las carreras de PB y LB que se anotaron para cursar QGI en el primer año de ambas carreras. El número total de alumnos fue de 40 .

\section{Instrumentos}

Los instrumentos aplicados fueron los siguientes:

a) Cuestionario según escala Likert: En este instrumento se usa una escala que va desde el total desacuerdo (TD) hasta el total acuerdo (TA). Para cuantificar las respuestas se asigna un valor a cada opción de la escala Likert, de 1 a 5 para las preguntas que están en forma positiva, así: TA (en total acuerdo) $=5, A$ (acuerdo) $=4$, I (indecisión) $=3$, $D($ desacuerdo $)=2$ y TD (en total desacuerdo) $=1$; mientras que para las preguntas en forma negativa la escala se invierte. El cuestionario utilizado fue adaptado de Molina, Carrizo y Farías (2011). Se realizaron varias pruebas piloto que permitieron que el instrumento utilizado en forma definitiva fuera de alta fiabilidad, con un alfa de Cronbach de 0,81 . Este índice es clasificado como muy bueno por Altman (1990). El cuestionario completo se observa en el anexo i. El mismo constó de 18 proposiciones declarativas (PD), 10 redactadas de forma positiva y 8 de forma negativa, buscando evitar tendencias al responder. Las preguntas están distribuidas en cuatro categorías o dimensiones: importancia de la química y del curso de química (PD: 4, 7, 9 y 14); dificultad en el estudio y comprensión de la química (PD: 2, 5, 11, 12 y 17); interés por el curso de química (PD: 1, 3, 6, 10, 13, y 16) y utilidad del conocimiento químico (PD: 8 , 15 y 18). Para la técnica de Likert se consideran actitud positiva las respuestas con puntuación 4 y 5; indiferente: puntuación 3 y actitud negativa: puntuación 1 y 2 . Teniendo en cuenta lo anterior, y siguiendo a Pliego, Contini, Odetti, Güemes y Ti- 
burzi (2004), a los efectos de asignar categorías de "valoración" a las respuestas obtenidas para las diferentes PD se aplicó el siguiente criterio de división en subintervalos, según la puntuación media (PM) de cada PD: valoración negativa:

$(1,00<\mathrm{PM} \leq 2,33)$; valoración indiferente: $(2,34<$ $\mathrm{PM} \leq 3,67)$ y valoración positiva:

$(3,68<\mathrm{PM} \leq 5,00)$. En el caso de las PD formuladas en forma negativa $(2,6,8,10,11,13,16$ y 18), esta escala de subintervalos se invierte: positiva $(1,00<\mathrm{PM} \leq 2,33)$; indiferente

$(2,34<\mathrm{PM} \leq 3,67)$ y negativa $(3,68<\mathrm{PM} \leq 5,00)$.

b) Cuestionario según el método de diferencial semántico: En este instrumento se plantean adjetivos y/o situaciones contrarias que el alumno debe puntuar de 0 a 10. El cuestionario fue adaptado de Odetti et al. (2009) con 10 ítems y del mismo modo que para el método L, también para DS se realizó una prueba piloto con el objetivo de obtener un instrumento confiable. En este caso el valor del índice alfa de Cronbach fue de 0,83 (anexo ii). Al igual que en el instrumento anterior y a los efectos de asignar categorías de "valoración" a las respuestas obtenidas para los adjetivos y/o situaciones contrarias se aplicó el siguiente criterio de división en subintervalos de valoración: negativa: $(0,00<\mathrm{PM} \leq 3,50)$, indiferente; $(3,51<\mathrm{PM} \leq$ $6,50)$ y positiva

$(6,51<P M \leq 10,00)$, siguiendo lo propuesto por Pliego et al. (2004).

\section{Análisis estadístico}

Para el análisis estadístico de los resultados se utilizó el programa spss (Statistical Package for the Social Sciences). La fiabilidad de los instrumentos cuantitativos (L y DS) fue obtenida a través del coeficiente alfa de Cronbach. Para determinar si las técnicas de $L$ y DS son equiparables se calculó el índice kappa que mide la concordancia entre los instrumentos antes mencionados con un nivel de significancia del $5 \%$.

\section{Resultados y discusión}

En la tabla 1 se presentan los resultados obtenidos a partir de la aplicación del cuestionario L. En la misma pueden observarse los valores de PM obtenidos a partir de cada PD con su correspondiente desviación estándar y subintervalo de valoración, donde se advierte un gran número de actitudes valoradas como indiferentes. Es importante destacar que no se registraron actitudes negativas. El alto porcentaje de actitudes indiferentes registradas por el cuestionario $L$ puede deberse a que los encuestados son alumnos ingresantes al sistema universitario, que hace pocos meses han egresado del último año del nivel secundario o medio, donde según Espinosa García y Román Galán (1998), la apatía hacia la química se refleja con mayor fuerza justamente en estudiantes de los últimos años del nivel medio. Sin embargo, las dos PD calificadas como positivas: "La química nos ayuda a comprender muchos aspectos de la vida cotidiana" (PD n. ${ }^{\circ} 4$ del cuestionario L), correspondiente a la categoría "importancia de la química y del curso de química" y fundamentalmente la PD n. ${ }^{\circ}$ 15: "Los conocimientos en química son necesarios para el desarrollo de mi carrera", la cual forma parte de la dimensión "utilidad del conocimiento químico", constituyen sin dudas elementos facilitadores del aprendizaje. 
Tabla 1. Puntuaciones medias (PM) con su respectiva variación estándar para alumnos que cursaron Química General e Inorgánica según el método L

\begin{tabular}{|c|c|c|c|c|c|}
\hline Ítem & PM & Actitud & Ítem & PM & Actitud \\
\hline 1 & $7,05 \pm 2,12$ & Positiva & 6 & $6,90 \pm 1,72$ & Positiva \\
\hline 2 & $6,00 \pm 2,36$ & Indiferente & 7 & $7,40 \pm 1,66$ & Positiva \\
\hline 3 & $5,68 \pm 2,57$ & Indiferente & 8 & $6,75 \pm 2,03$ & Positiva \\
\hline 4 & $6,30 \pm 2,97$ & Indiferente & 9 & $5,55 \pm 2,55$ & Indiferente \\
\hline 5 & $7,73 \pm 2,28$ & Positiva & 10 & $8,00 \pm 1,80$ & Positiva \\
\hline
\end{tabular}

Los valores promedios con su respectiva desviación estándar e intervalo de valoración para los resultados obtenidos según el método de DS, se presentan en la tabla 2. En la misma se aprecia que la técnica de DS registra menor número de actitu- des valoradas como indiferentes y un mayor número de actitudes valoradas como positivas comparativamente con la escala L. Al igual que para el cuestionario L, no se contabilizaron actitudes hacia la química valoradas como negativas.

Tabla 2. Puntuaciones medias (PM) con su respectiva variación estándar para alumnos que cursaron Química General e Inorgánica según el método DS

\begin{tabular}{|c|c|c|c|c|c|}
\hline PD & PM & Actitud & PD & PM & Actitud \\
\hline 1 & $2,80 \pm 1,07$ & Indiferente & 10 & $2,70 \pm 1,09$ & Indiferente \\
\hline 2 & $2,98 \pm 1,19$ & Indiferente & 11 & $3,57 \pm 1,03$ & Indiferente \\
\hline 3 & $2,40 \pm 0,87$ & Indiferente & 12 & $2,37 \pm 1,02$ & Indiferente \\
\hline 4 & $3,88 \pm 0,99$ & Positiva & 13 & $3,65 \pm 1,27$ & Indiferente \\
\hline 5 & $2,82 \pm 1,10$ & Indiferente & 14 & $2,55 \pm 0,88$ & Indiferente \\
\hline 6 & $2,98 \pm 1,19$ & Indiferente & 15 & $4,05 \pm 0,89$ & Positiva \\
\hline 7 & $2,36 \pm 0,99$ & Indiferente & 16 & $3,20 \pm 0,46$ & Indiferente \\
\hline 8 & $2,90 \pm 0,93$ & Indiferente & 17 & $2,35 \pm 0,87$ & Indiferente \\
\hline 9 & $2,95 \pm 0,84$ & Indiferente & 18 & $3,07 \pm 0,97$ & Indiferente \\
\hline
\end{tabular}


A los efectos de decidir si los instrumentos de medición concuerdan como para que uno reemplace al otro, se confrontaron ambos a través de una tabla de contingencia (tabla 3) y se determinó cuántos acuerdos exactos ocurrieron. La totalidad de alumnos asciende a 40 y mediante el cuestionario $\mathrm{L}$ se detectaron 38 alumnos con actitudes indiferentes y 2 alumnos con actitud positiva, mientras que el método de DS detectó 19 personas con actitudes indiferentes y 21 con actitudes valoradas como positivas. Existen 19 casos en que se detectaron actitudes indiferentes por los dos métodos y 2 casos en los que se observaron actitudes positivas tanto por el método de DS como por la técnica L. También es importante destacar que hay discordancias, ya que 19 alumnos manifesta- ron una actitud positiva mediante el DS mientras que tienen actitud indiferente para L. Es decir, si las escalas concordaran totalmente, fuera de las celdas sombreadas de la tabla 3, debería haber ceros. Por lo tanto, los resultados obtenidos por la escala Likert no son replicados por el método de DS. Una medida estadística de la concordancia cuando se tienen variables de tipo categórico es el índice o coeficiente kappa de Cohen (K). Para Altman (1990) el valor o del coeficiente se presenta cuando hay mayor desacuerdo y un valor de $1 \mathrm{im}$ plica mayor acuerdo entre instrumentos. El valor del coeficiente $\mathrm{K}$ en el presente trabajo fue 0,091 (prácticamente cero) lo cual nos indica que no hay concordancia entre los instrumentos.

Tabla 3. Tabla de contingencia que muestra el grado de acuerdo entre las actitudes según los cuestionarios L y DS

\begin{tabular}{|c|c|c|c|}
\hline \multirow{2}{*}{ LIKERT } & \multicolumn{2}{|c|}{ DIFERENCIAL SEMÁNTICO } & \\
\cline { 2 - 4 } & Actitud indiferente & Actitud positiva & Total \\
\hline Actitud indiferente & 19 & 19 & 38 \\
\hline Actitud positiva & 0 & 2 & 2 \\
\hline Total & 19 & 21 & 440 \\
\hline
\end{tabular}

Los resultados obtenidos en este trabajo difieren de los hallados por Espinosa García y Román Galán (1998), ya que estos concluyeron que ambas técnicas de evaluación de actitudes proporcionan resultados equiparables en el sistema educativo español al analizar la actitud hacia las ciencias experimentales en general. Pero sí son coincidentes con los resultados a los cuales arriban Odetti et al. (2009), quienes obtuvieron coeficientes kappa iguales a 0,0087 y de 0,0184 al evaluar las actitudes hacia la química en estudiantes de las carreras de Bioquímica y Licenciatura en Nutrición respectivamente, comparando los cuestionarios de L y DS.

\section{Conclusiones}

Ninguno de los dos instrumentos registró actitudes hacia la química valoradas como negativas, lo cual es un dato esperanzador para el proceso de enseñanza-aprendizaje de esta asignatura y brinda conocimientos elementales que serán aplicados en materias correlativas como Química Orgánica, Química Biológica, Biología Celular y Molecular, entre otras. Sin embargo, se observaron diferencias en la medición de las actitudes valoradas como indiferentes y positivas, esto es consecuencia de que los métodos aplicados no miden en forma equiparable el objeto de medición, y nuestra 
conclusión es que no son intercambiables, pues no se obtienen los mismos resultados con ambos instrumentos (índice kappa prácticamente cero), ya que el DS utilizado no tiene en cuenta la totalidad de categorías o dimensiones establecidas en el cuestionario según la escala Likert para este trabajo (importancia de la química y del curso de química — dificultad en el estudio y comprensión de la química - interés por el curso de química y utilidad del conocimiento químico). Se podría validar un nuevo cuestionario para el método de diferencial semántico que incluya todas las dimensiones de análisis antes mencionadas.

\section{Referencias}

Altman, D. G. (1990). Practical Statistics for Medical Research. Londres: CRC Press.

Bauer, C. F. (2008). Attitude towards Chemistry: A Semantic Differential Instrument for Assessing Curriculum Impacts. Journal of Chemical Education, 85(10), 1440-1445.

Duit, R. y Treagust, D. F. (2012). Teaching Science for Conceptual Change; Theory and Practice. En B. J. Fraser, K. G. Tobin y C. J. Mc Robbie (Ed.), International Handbook of Science Education. Dordrecht: Springer (pp. 19-93).

Espinosa García, J. y Román Galán, T. (1998). La medida de las actitudes usando las técnicas de Likert y de Diferencial Semántico. Enseñanza de las Ciencias, 16(3), 477-484.

Garritz, A., Rueda, C., Robles, C. y Vázquez-Alonso, A. (2011). Actitudes sobre la naturaleza de ciencia y tecnología en profesores y estudiantes mexicanos del bachillerato y la universidad públicos. Educación Química, 22(2), 141-154.

Molina, M., Carrizo, J. y Farías, D. (2011). Actitudes hacia la química de estudiantes de diferentes carreras universitarias en Colombia. Química Nova, 34(9), 1672-1677.

Nieswandt, M. (2007). Student Affect and Conceptual Understanding in Learning. Journal of Research in Science Teaching, 44 (7), 908-937.
Odetti, H., Tiburzi, M., Mondino, A. y Güemes, R. O. (2009). Likert y Diferencial Semántico en la medición de actitudes hacia la química en estudiantes universitarios. Aula Universitaria, 11, 69-82.

Pliego, O. H., Contini, L., Odetti, H., Güemes, R. O. y Tiburzi, M. (2004). Las actitudes de los estudiantes universitarios hacia el fenómeno radiactivo, la energía nuclear y sus aplicaciones. Educación Química, 15(2), 60-66.

Venturini, P. (2004). Attitudes des éléves envers les sciencies: le point des recherché. Revue Française de Pédagogie, 149, 125-143.

Xu, X. y Lewis J. (2011). Refinement of Chemistry Attitude Measure for College Students. Chemical Education, 88, 561-568. 


\section{Anexo I. Cuestionario según escala Likert}

Estos cuestionarios han sido diseñados para conocer tus actitudes hacia la Química y mejorar la calidad de la enseñanza de esta asignatura, que te brindará elementos básicos para desarrollarte en la carrera que elegiste. Si querés colaborar con esta investigación, te garantizo que tu participación será anónima, reservada y confidencial. Es importante que sepas que tus respuestas

NO AFECTARÁN EN ABSOLUTO TUS CALIFICACIONES COMO ESTUDIANTE. Desde ya muchas gracias por colaborar.

En esta encuesta hay que marcar una sola opción. Las opciones de respuestas son las siguientes:

TA = totalmente de acuerdo. $A=$ de acuerdo. $I=$ Indecisión. $D=$ en desacuerdo. $T D=$ totalmente en desacuerdo.

\begin{tabular}{|c|l|c|c|c|c|c|}
\hline \multicolumn{7}{|c|}{ CARRERA: ...................CUATRIMESTRE................. AÑo............... SEXO: F / M } \\
\hline 1 & La química me gusta más que otras materias & TA & A & I & D & TD \\
\hline 2 & La simbología utilizada en las clases de química me parece difícil de entender & TA & A & I & D & TD \\
\hline 3 & Me gustaría tener mayor carga horaria de química & TA & A & I & D & TD \\
\hline 4 & La química nos ayuda a comprender muchos aspectos de la vida cotidiana. & TA & A & I & D & TD \\
\hline 5 & Resuelvo con facilidad los ejercicios de química & TA & A & I & D & TD \\
\hline 6 & Me aburro en las clases de química & TA & A & I & D & TD \\
\hline 7 & La esperanza en resolver muchos problemas ambientales está en la química & TA & A & I & D & TD \\
\hline 8 & Mi desempeño profesional es independiente del conocimiento químico. & TA & A & I & D & TD \\
\hline 9 & El progreso del país está relacionado con el avance de la química. & TA & A & I & D & TD \\
\hline 10 & La química es una ciencia muy compleja para mi nivel de conocimiento & TA & A & I & D & TD \\
\hline 11 & Debo esforzarme mucho para aprender química & TA & A & I & D & TD \\
\hline 12 & El lenguaje de la química y sus símbolos son fáciles de entender. & TA & A & I & D & TD \\
\hline 13 & La actividad de un químico es poco interesante & TA & A & I & D & TD \\
\hline 14 & Todas las carreras universitarias deberían tener química en su currículo. & TA & A & I & D & TD \\
\hline 15 & Los conocimientos en química son necesarios para el desarrollo de mi carrera & TA & A & I & D & TD \\
\hline 16 & $\begin{array}{l}\text { Desearía que el programa de mi carrera tuviera menos asignaturas relacionadas } \\
\text { con la química. }\end{array}$ & TA & A & I & D & TD \\
\hline 17 & Comprendo los conceptos de química con facilidad & TA & A & I & D & TD \\
\hline 18 & Consideraría cambiarme de carrera y elegir otra donde no hubiera química & TA & A & I & D & TD \\
\hline
\end{tabular}




\section{Anexo Il. Cuestionario según el método de diferencial semántico}

Estos cuestionarios han sido diseñados para conocer tus actitudes hacia la Química y mejorar la calidad de la enseñanza de esta asignatura, que te brindará elementos básicos para desarrollarte en la carrera que elegiste. Si querés colaborar con esta investigación, te garantizo que tu participación será anónima, reservada y confidencial. Es importante que sepas que tus respuestas NO AFECTARÁN EN ABSOLUTO TUS CALIFICACIONES COMO ESTUDIANTE.

Desde ya muchas gracias por colaborar.

A continuación se expresan una serie de adjetivos o situaciones contrarias referidas a la disciplina QUÍMICA. Señala con una puntuación entre 0 y 10 cada una de ellas. Si crees que la cuestión se identifica con lo expuesto a la izquierda redondea el 10, si con la derecha el 0 . Si es una situación intermedia, según su proximidad a una u otra situación, acercate más o menos en la puntuación a uno de los extremos.

\begin{tabular}{|c|c|c|}
\hline 1. Interesante & 109876543210 & Aburrido \\
\hline 2. Claro & 109876543210 & Confuso \\
\hline 3. Fácil & 109876543210 & Difícil \\
\hline 4. Me acercó a la naturaleza & 109876543210 & $\begin{array}{l}\text { No me acercó a la natura- } \\
\text { leza }\end{array}$ \\
\hline 5. Probaría otra vez & 109876543210 & No probaría otra vez \\
\hline 6. Accesible & 109876543210 & Imposible \\
\hline 7. Aprendí & 109876543210 & No aprendí \\
\hline 8. Me gustó & 109876543210 & No me gustó \\
\hline 9. Me divertí & 109876543210 & Sufrí \\
\hline 10. Valía la pena & 109876543210 & Tiempo perdido \\
\hline
\end{tabular}

